\title{
Post-Stroke Cognitive Impairment: Pathophysiological Insights into Brain Disconnectome from Advanced Neuroimaging Analysis Techniques
}

\author{
Jae-Sung Lim, ${ }^{\mathrm{a}, *}$, Jae-Joong Lee, ${ }^{\mathrm{b}, \mathrm{c}, *}$, Choong-Wan Woo ${ }^{\mathrm{b}, \mathrm{c}, \mathrm{d}}$

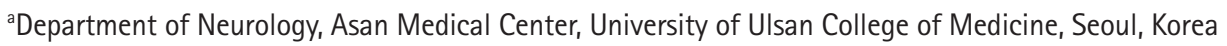 \\ ${ }^{b}$ Department of Biomedical Engineering, Sungkyunkwan University, Suwon, Korea \\ 'Center for Neuroscience Imaging Research, Institute for Basic Science, Suwon, Korea \\ ${ }^{\mathrm{d}}$ Department of Intelligent Precision Healthcare Convergence, Sungkyunkwan University, Suwon, Korea
}

The neurological symptoms of stroke have traditionally provided the foundation for functional mapping of the brain. However, there are many unresolved aspects in our understanding of cerebral activity, especially regarding high-level cognitive functions. This review provides a comprehensive look at the pathophysiology of post-stroke cognitive impairment in light of recent findings from advanced imaging techniques. Combining network neuroscience and clinical neurology, our research focuses on how changes in brain networks correlate with post-stroke cognitive prognosis. More specifically, we first discuss the general consequences of stroke lesions due to damage of canonical resting-state large-scale networks or changes in the composition of the entire brain. We also review emerging methods, such as lesion-network mapping and gradient analysis, used to study the aforementioned events caused by stroke lesions. Lastly, we examine other patient vulnerabilities, such as superimposed amyloid pathology and blood-brain barrier leakage, which potentially lead to different outcomes for the brain network compositions even in the presence of similar stroke lesions. This knowledge will allow a better understanding of the pathophysiology of post-stroke cognitive impairment and provide a theoretical basis for the development of new treatments, such as neuromodulation.

Keywords Stroke; Cognitive dysfunction; Dementia; Neuroimaging; Brain mapping; Connectome
Correspondence: Jae-Sung Lim Department of Neurology, Asan Medical Center, University of Ulsan College of Medicine, 88 Olympic-ro 43-gil, Songpagu, Seoul 05505, Korea Tel: +82-2-3010-3427 Fax: +82-2-474-4691 E-mail: jaesunglim@amc.seoul.kr https://orcid.org/0000-0001-6157-2908

Co-correspondence: Choong-Wan Woo Department of Biomedical Engineering, Sungkyunkwan University, 2066 Seoburo, Jangan-gu, Suwon 16419, Korea Tel: +82-31-299-4363

Fax: +82-31-299-4506

E-mail:waniwoo@g.skku.edu https://orcid.org/0000-0002-7423-5422

Received: July 4, 2021

Revised: September 17, 2021

Accepted: September 17, 2021

*These authors contributed equally to the manuscript as first author.

\section{Introduction}

Traditionally, neurological symptoms of stroke have provided a significant basis for understanding the functional mapping of the brain. Studies by Wilder Penfield, Paul Broca, and Carl Wernicke have led to a better understanding of various high-level cognitive functions, and the localization of the areas involved in these processes across the brain. ${ }^{1-3}$ Carl Wernicke suggested that functions such as language, motor, and primary sensory domains are relatively well localized, while high-level cortical functions, such as memory and execution, seem to depend on the communication among several areas of the brain rather than on a specific region. ${ }^{2}$ A century later, Wernicke's hypothesis was confirmed through advanced neuroimaging techniques and various computational neuroscience methods.

Post-stroke cognitive impairment (PSCl) is defined as the occurrence of any type of cognitive deterioration after stroke, ranging from minor impairment to dementia. ${ }^{4} \mathrm{PSCl}$ has been 
considered a unique clinical entity, distinct from Alzheimer's disease or subcortical vascular cognitive impairment. As shown by Penfield's experiments and Wernicke's lesion study, $\mathrm{PSCl}$ is a valuable disease model that can be considered as a "brain lesion-cognitive disorder." It has gradually evolved from past experimental studies of patients with specific symptoms that revealed the underlying neural substrates, and a great body of related knowledge has been accumulated through lesionsymptom mapping methods. Location of the stroke lesion is one of the most important determinants of the cognitive outcome $^{5}$ and collecting lesion data from patients with specific symptoms provides information about the neural substrates affected in each specific case. ${ }^{6,7}$ In recent studies, lesion-symptom mapping has been used to identify the neural substrates of particular cognitive functions, ${ }^{8-10}$ or predict the prognosis of post-stroke functional deficits, such as aphasia. ${ }^{11}$ It was also used to study the neural substrates of emotional disorders, such as post-stroke depression, and to understand the inherent limitation of certain cognitive assessment tools. ${ }^{12}$ Moreover, lesion-symptom mapping studies have shown that small vessel disease (SVD), as well as stroke lesions, can additionally cause a decrease in the processing speed if distributed in the frontal white matter. ${ }^{6}$ However, not all neurological symptoms can be localized using lesion-symptom mapping, as the causative relationship between lesion and cognitive impairment is not universally applicable. In example, the explanatory power of regression model $\left(R^{2}\right)$ was 0.35 to 0.58 for motor and visual functions, while limited to 0.06 for verbal memory..$^{13}$

Fox ${ }^{14}$ suggested that a network perspective could be a significant breakthrough in overcoming the limitations of lesionsymptom mapping by showing that there is a network connecting the lesions at various locations causing the same behavioral symptoms. Functional magnetic resonance imaging (fMRI) and diffusion tensor imaging (DTI) have enabled researchers to apply this network approach. In addition, recent advances in imaging have also provided insights into underlying vulnerabilities, such as amyloid pathology, SVD, and bloodbrain barrier (BBB) disruption, which lead to different cognitive prognosis even in the case of the same stroke lesions.

In this review, we provide a comprehensive look at the pathophysiology of $\mathrm{PSCl}$ in light of recent research using advanced imaging techniques. Recent developments in this field are expected to deepen our understanding of the functional localization of brain areas and provide important information on the targets of various neuromodulation techniques. Going one step further from the size or localization of lesions that can be observed with conventional imaging techniques, we focus on how the qualitative and quantitative changes in the composition of the underlying brain networks can be visualized, quantified, and correlated with post-stroke cognitive prognosis. Furthermore, neuroimaging research focusing on amyloid and BBB leakage is important in understanding the adversarial environment caused by underlying diseases, independent of stroke. These efforts will serve as an important foundation for screening groups at high risk of PSCl, developing appropriate neuromodulation therapy, and determining the treatment outcomes.

\section{Remote effects throughout the brain}

Classical studies, including those discussed above, are based on the assumption that stroke lesions cause symptoms by disrupting the function of the corresponding local brain regions. However, due to recent advances in neuroimaging techniques, it has gradually been accepted that the effects of stroke lesions are not confined to the local lesion site. The classical concept of "diaschisis" is a good example of the remote effects of lesions. ${ }^{15}$ The concept of remote lesion effects is particularly useful when studying the relevance of stroke lesions to cognitive outcomes. For example, it is easy to predict that if a stroke lesion occurs in the hand knob area, paralysis in the contralateral hand will occur. However, it is not intuitively understood how and why stroke lesions in the anterior thalamic radiation result in cognitive impairment. Lesions in the anterior thalamic radiation sever structural connections to the frontal lobe, reducing the frontal cortical thickness, and leading to impairment in frontal cortical functions, including processing speed and executive function. ${ }^{6,16}$ If stroke lesions occur in the hub regions of the well-known intrinsic functional network, their effects can reach a wide range of areas of the brain that are functionally connected, and not only the perilesional areas. Alstott et al. ${ }^{17}$ visualized this system-wide effect by lesions in the hubs through computational modeling. Clinical studies have also reported that cognitive impairment is more likely to arise when lesions occur in the hub region, which mainly corresponds to the heteromodal association cortex. ${ }^{18,19}$

There are two types of brain connectivity: functional connectivity, which can be measured by functional neuroimaging techniques, such as resting-state fMRI (rs-fMRI); and structural connectivity, which can be measured by structural neuroimaging techniques, such as DTI. Research using DTI infers structural connectivity through white matter tracts and covers several areas. Analysis methods for DTI studies range from tractography and tract-based spatial statistics to network models using graph theory. ${ }^{20,21}$ A recent study using DTI compiled an "Atlas of white matter function" based on 1,333 stroke lesions (available from 
https://identifiers.org/neurovault.collection:7735). ${ }^{22}$ It has been shown that a "stroke disconnectome" that comprehensively considers the effects of structurally interconnected regions in the brain, rather than only the location of the stroke lesion, better describes the cognitive prognosis of patients with subcortical stroke. These results emphasize that subcortical lesions can also lead to a decline in higher cognitive functions, which were traditionally thought to be the exclusively caused by cortical lesions, and that the disruption of white matter connectivity can be an important mechanism underlying this phenomenon. On the other hand, network studies using rs-fMRI have identified that key brain regions can be functionally affected by lesions even if they are not structurally connected..$^{13,14}$ In recent years, an "indirect lesion-network mapping" methodology has been proposed to study the network-wide effects of local lesions by applying the normative functional connectivity maps obtained from healthy individuals to the patient's lesion location without directly taking rs-fMRls from stroke patients. ${ }^{14}$

In the following sections, we introduce these approaches from a clinical perspective and discuss the main considerations derived from each representative study. Network analysis in stroke research can be classified into two main perspectives. The first is to examine how stroke lesions result in changes in the intrinsic brain networks and how these changes relate to cognitive prognosis. The second is to look at the brain as a single integrated system and determine how the composition of this system changes and how such changes relate to cognitive function. In this review, we first focus on the role of brain connectivity in the pathophysiology of $\mathrm{PSCl}$ from a reductionist perspective, considering changes in several representative large-scale brain networks. We then move to a more holistic perspective, analyzing the brain network as a complex system. Lastly, we discuss the underlying vulnerabilities that can lead to differences in network configurations between patients with the same lesions.

\section{From part to whole (I): a reductionist perspective}

$\mathrm{fMRI}$ is a common research tool used to identify neural substrates by administering a certain task to an individual and analyzing the resulting signal changes. Thus, it has been the foundation of cognitive neuroscience research. However, it is not easy to analyze complex cognitive tasks during functional imaging in patients with stroke sequelae. From this perspective, the analysis of fMRI signals from the resting state, without the assignment of any specific task, has clinical utility. Previous studies suggested that network activity was consistently observed during the resting state, and the corresponding networks were called resting-state networks (RSNs). ${ }^{23}$ These RSN include default mode, central executive, dorsal attention, and salience networks. ${ }^{24}$ Typical canonical networks have been studied in a variety of fields, such as normal aging, schizophrenia, depression, pain, and Alzheimer disease. ${ }^{25,26} \mathrm{~A}$ comprehensive review of the association between cognition and RSNs goes beyond the scope of this manuscript; thus, we recommend that the readers refer to recent review papers. ${ }^{27-29}$

With regard to RSNs, a previous study, that applied lesion mapping methods, has shown that the involvement of major hubs within the default mode network and the central executive network increases the risk of developing post-stroke global cognitive impairment, ${ }^{19}$ while another study directly examined changes in the activity of those hubs using rs-fMRI data in patients with stroke. ${ }^{30}$ These hubs include the medial temporal and hippocampal regions, which are already known as strategic regions, and the posterior parietal cortex, which is the heteromodal association cortex, as suggested by computational modeling ${ }^{17}$ and principal component analysis of connectivity hierarchy. ${ }^{31}$

Siegel et al. ${ }^{32}$ analyzed the communication between RSNs, as well as the activities of individual RSNs. Resting-state fMRI was performed in 132 patients with stroke and 31 matched controls, along with comprehensive cognitive evaluations at 1 to 3 weeks after stroke. Moreover, stroke consequences on 13 canonical RSNs and their relationships with cognitive prognosis were analyzed. They found that primary motor and visionrelated functions were dependent on specific RSNs, but higher cognitive functions, such as attention and memory, were more dependent on the communication between several RSNs than on the function of any individual RSN (Figure 1). Wernicke's prediction, quoted earlier, was proven using advanced neuroimaging techniques 100 years later.

In summary, lesions in each region of the brain have differential effects on cognitive functions, and these differences should be understood from a network perspective, rather than focusing only on the location of the lesion itself. Damage to the highly connected hubs within each network, or changes in communication between RSNs, were associated with the occurrence of PSCl. However, other studies on PSCl have attempted to explain the mechanisms of cognitive decline after stroke as a change in the overall structure and information transfer efficiency of the brain rather than applying the RSN perspective. In the next section, we discuss this second approach.

\section{From part to whole (II): brain as a complex system-a holistic perspective}

In addition to the RSN-based approach described above, imaging analysis techniques can model the brain as a single complex system. For example, the graph theory is a mathematical 

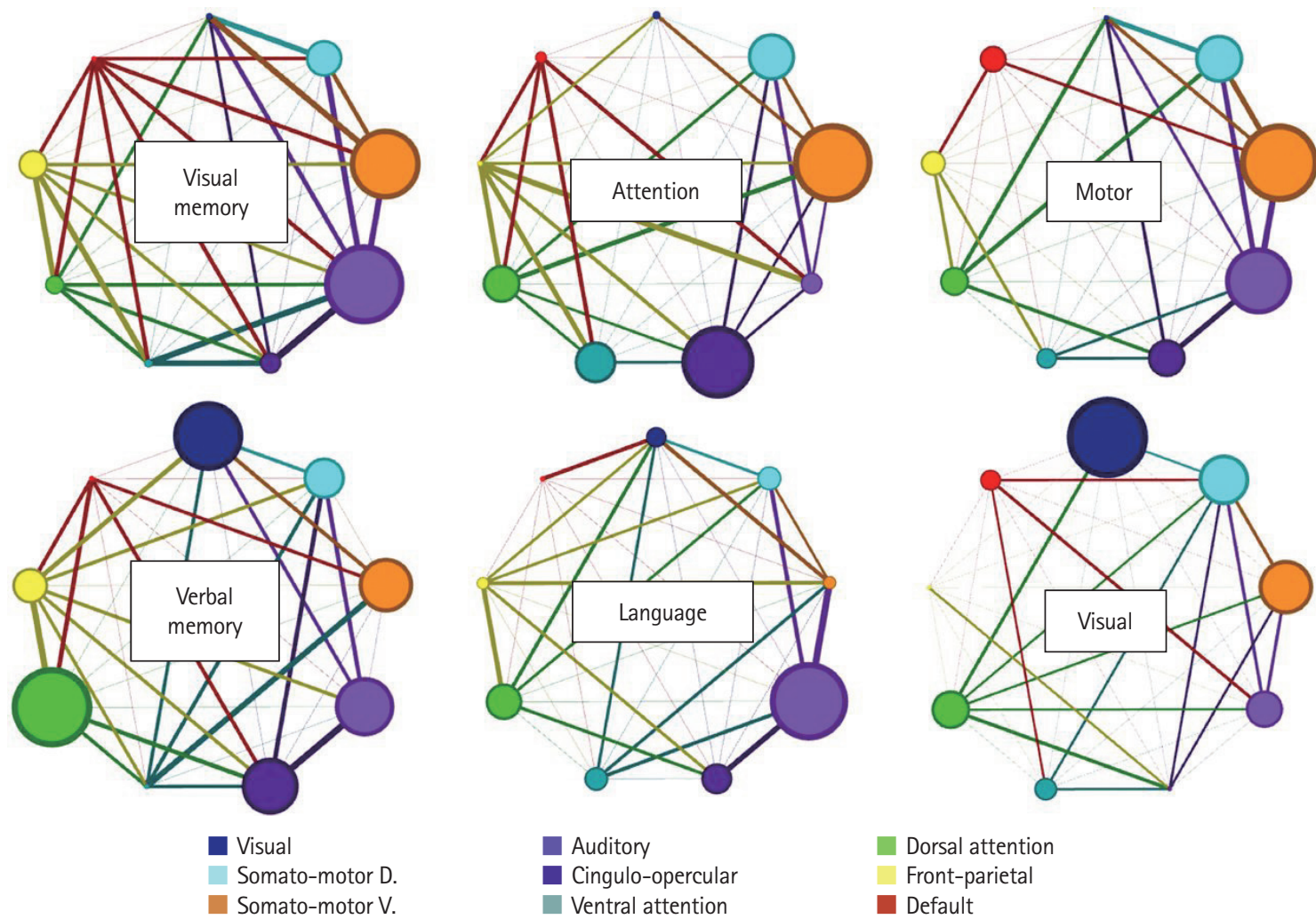

Dorsal attention

Front-parietal

Default

Figure 1. Contribution of functional connections within a resting-state network and between resting-state network pairs for each functional connectivity-behavior prediction. Each circle refers to a representative resting-state network, and its size is proportional to the contribution of within-network connections. Line thickness is proportional to the weighting of between-network connectivity. Adapted from Siegel et al., ${ }^{32}$ with permission from National Academy of Sciences.

approach that allows us to represent a complex network system in the form of simple lines (i.e., edges) and dots (i.e., nodes) and to extract some representative quantities that can reflect its characteristics. ${ }^{33}$ Graph theory has been used not only in neuroscience, but also in various other disciplines, such as social science and genetic association. For more information on graph theory, please refer to the review articles and books by Sporns, ${ }^{27}$ Rubinov and Sporns, ${ }^{33}$ and Bullmore and Sporns. ${ }^{34}$ In this section, we discuss the pathogenesis of PSCl through studies that have applied this holistic perspective.

\section{Network attributes and post-stroke cognition} Integration and segregation are important concepts in understanding the brain as a network system (Figure 2A). ${ }^{33}$ Integration is defined as "the ability to rapidly combine specialized information from distributed brain regions," and characteristic path length and global efficiency are well-known indicators of integration. ${ }^{33}$ Segregation, defined as "the ability for specialized processing to occur within densely interconnected groups of brain regions," is an indicator that quantifies the functional units within a network. ${ }^{33}$ Representative attributes of segregation include clustering coefficient, transitivity, and modularity.
In addition to these quantification measures, several methods to directly visualize functionally compartmentalized community structures have also been proposed. ${ }^{35,36}$ In brief, segregation means that functions such as language and vision are confined to a particular group of brain regions, while integration refers to the ability of these areas to communicate with each other more comprehensively from a brain-wide perspective. Figure $2 \mathrm{~B}$ shows how stroke lesions can lead to changes in integration and segregation. In the following sections, modularity, which is closely related to $\mathrm{PSCl}$, is discussed in detail.

Modules, modularity, and system segregation: an indicator for functional segregation

A module represents a functionally compartmentalized unit in the brain. ${ }^{33}$ As such, modularity is an indicator of the fact that functionally related brain regions exhibit highly correlated activity within the network and less correlated activity with other networks. This is a known strategy used by the brain to efficiently process the flow of information and maintain the brain functions that the network is responsible for. ${ }^{37}$ Figure $2 \mathrm{~A}$ and $\mathrm{B}$ show the relationship between the module and hub, and how focal lesions, such as strokes, change the cerebral network 

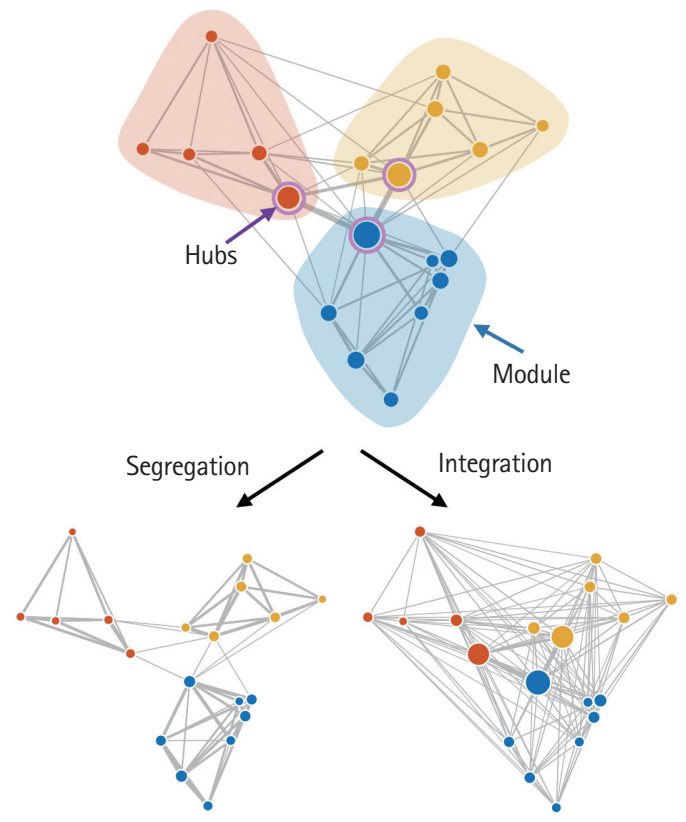

A
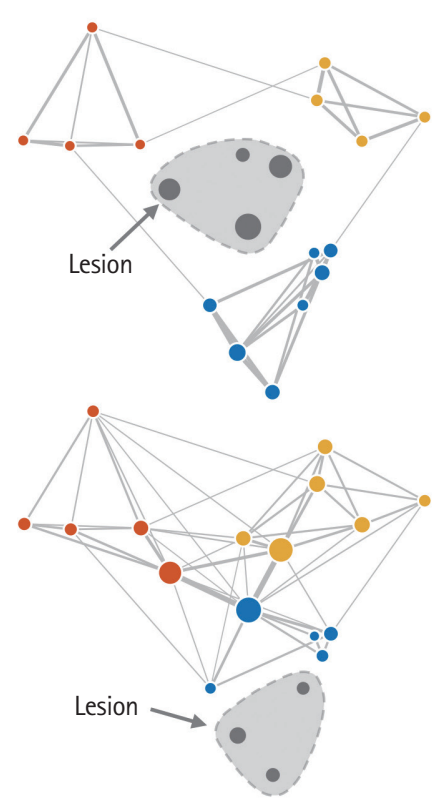

B

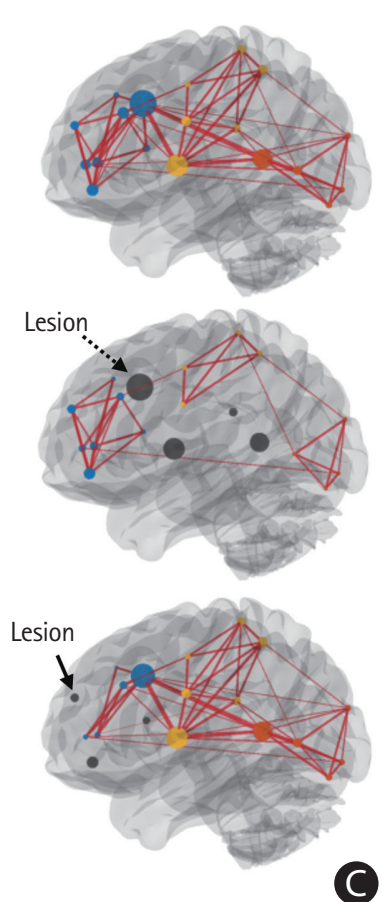

Figure 2. Integration and segregation in the brain as a complex system. (A) Schematic representation of functional segregation and integration. (B) Stroke lesions can cause different changes in segregation and integration depending on where they occur: upper panel, maintained segregation and reduced integration; bottom panel, decreased segregation while maintaining integration. (C) Stroke lesions can have widespread effects in the brain if they occur in the hubs (dashed arrow).

configuration. Stroke lesions can have different outcomes depending on the location of the lesion. In the case of the upper panel of Figure $2 \mathrm{~B}$, segregation is maintained, but integration is reduced as a consequence of decreased connectivity between functionally partitioned modules. However, as shown in the bottom panel of Figure $2 B$, a decrease in segregation can also occur without significant changes in integration. These changes can be widespread, disrupting the information flow not only in the peri-infarction area, but throughout the brain, and even in the contralesional hemisphere. ${ }^{38,39}$

Changes in these modules and hubs have been studied in relation to cognitive impairment after stroke. Siegel et al. ${ }^{36}$ performed an rs-fMRI study in stroke patients at 2 weeks (107 subjects), 3 months ( 85 subjects), and 1 year (67 subjects) after stroke. Modularity significantly decreased during the subacute period and then partially recovered during the subsequent period. In addition, the recovery of modularity led to the improvement of cognitive functions, such as language, spatial memory, and attention, but not to the recovery of motor and visual functions. In a task-based fMRI study of 15 patients with left hemispheric stroke with aphasia, the correlation between written language production accuracy and modularity was examined.$^{40}$ Although global integration was maintained, patients with poor local integrity and modularity had more severe symptoms and poor response to treatment. Separately, the modularity and local integrity of the central occipitaltemporal regions related to spelling function improved after treatment.

In addition, Chan et al. ${ }^{41}$ introduced the "system segregation" variable to quantify the degree of segregation of the brain. They demonstrated that changes in system segregation due to aging were significantly associated with episodic memory function. They suggested that "system segregation" can be a good indicator of higher cognitive function, as even younger subjects would display poor memory in the presence of low system segregation, while even the elderly displayed good memory function if they had high system segregation.

Although the changes in segregation due to stroke lesions were mostly interpreted from a pathological perspective, it was also suggested that these segregated brain structures could prevent the spread of damage caused by focal brain lesions. In other words, some researchers suggested an additional role of brain segregation in isolating brain damage within a specific module to minimize its impact, thereby preventing the overall deterioration of brain function. ${ }^{37,42}$

These studies, which are related to modularity, within-network connectivity, and system segregation, are good examples of how neuroplasticity occurs in the brain during post-stroke 

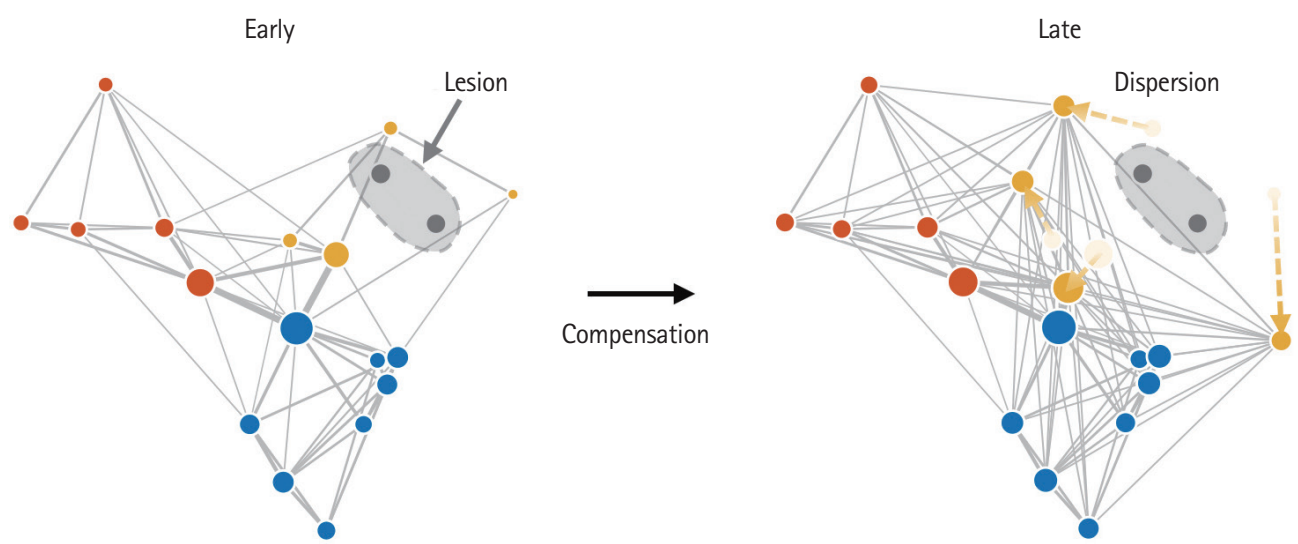

Figure 3. Dispersion caused by lesions and the subsequent compensation processes. The intra-network connectivity of the lesion-occurring module is weakened and dispersed; however, the compensation for this can enhance the between-network connectivity with functionally adjacent networks.

treatment, and suggest that advanced neuroimaging techniques, such as fMRI, can provide clinically useful information.

\section{Hubness: integration throughout the brain}

Along with changes in the composition of modules in the brain, the brain-wide effects of stroke lesions depend on the involvement of the hub regions, as illustrated in Figure 2C. Hubs play a crucial role in the flow of information between modules, which results in wider intracerebral functional changes when compromised. In the figure, lesions in the hubs (blue circle) have a more pronounced effect on the overall network configuration compared to those not involving hubs, in addition to the destruction of the module themselves (expressed in each color). ${ }^{39}$

Aben et al. ${ }^{43}$ quantified the hubness to study its association with PSCl. DTI and cognitive tests were performed in the 5th week after ischemic stroke in 75 patients, and cognitive tests were repeated 1 year later to evaluate recovery. The hub scores of gray matter regions, segmented into 90 regions on the brain network map, were obtained from 44 cognitively normal individuals. The proportion of volume of the region affected by the stroke lesion was calculated and multiplied by the hub score of the region, thus obtaining a "lesion impact score," that would index the influence of the lesion. This study showed that the lower the lesion impact score, the better the cognitive prognosis 1 year after stroke onset. Moreover, it underlined a negative correlation between the lesion impact score and global efficiency, demonstrating that a lesion invading a hub clearly affects the overall brain network integration.

\section{Interplay between segregation and integration}

The relationship between segregation and integration is also a significant issue. Previous studies have reported that function- ally segregated sensorimotor systems are loosened during the aging process. ${ }^{41}$ In the case of the cingulo-opercular control system, within-system connectivity decreases after middle age, but between-system connectivity increases, which is considered a compensatory change. In other words, once the densely segmented network becomes loose, it starts enhancing systemwide information transfer as a coping mechanism. In patients with stroke, the same process may occur during the recovery process after the lesion is formed. The intra-network connectivity of the lesioned module is weakened and dispersed; however, between-network connectivity with functionally adjacent networks can be increased (Figure 3). The initial decrease in modularity after brain damage (as described in section 'Modules, modularity, and system segregation: an indicator for functional segregation') is also in line with this pathological change. ${ }^{36}$ Poor prognosis may occur if these compensation mechanisms are not properly implemented. Guo et al. ${ }^{44}$ studied the imbalance between segregation and integration in 17 patients with post-stroke aphasia. In this study, both parameters were decreased compared to those of healthy controls. This disruption of intra-hemispheric networks in language-relevant regions without compensation was significantly correlated with the aphasia quotient. In addition, Wang et al. ${ }^{45}$ studied dynamic functional network connectivity using time-varying analysis methods in 58 patients with pontine stroke. They showed that patients with pontine stroke spent less time in a highly segregated state, which was associated with long-term low verbal memory scores. These results suggest that abnormal functional segregation and integration can also be observed in patients with infratentorial stroke. Furthermore, $\mathrm{Xu}$ et al. ${ }^{46}$ revealed that regional connectivity was disrupted in the cognitive and limbic systems in patients with post-stroke depression, while global efficiency was increased. The abnormal segregation-integration 


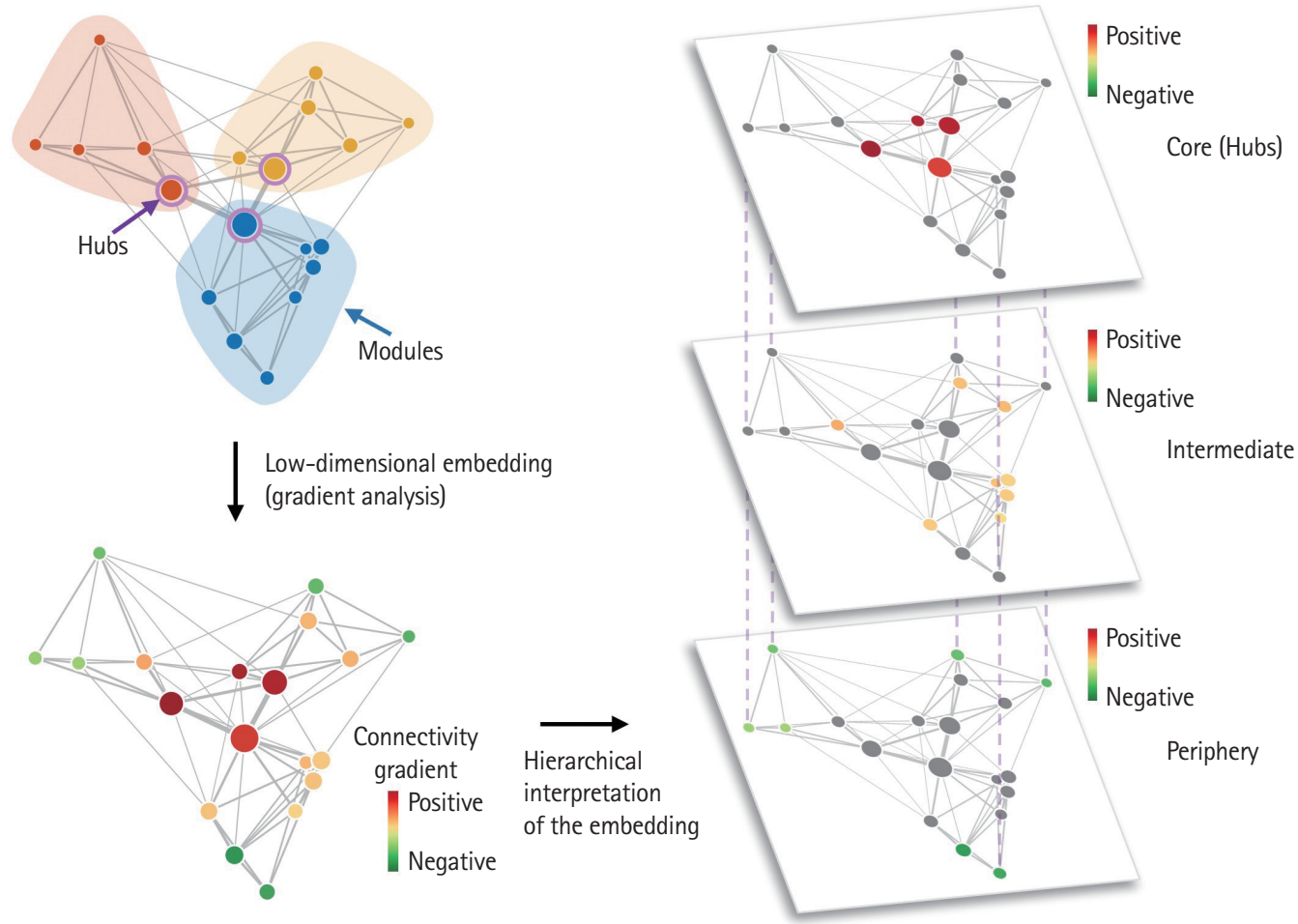

Figure 4. Hierarchy in brain connectivity. A hierarchy exists in functional brain connections. Figure $2 \mathrm{~A}$ can be represented according to the connectivity hierarchy via a gradient analysis. In the lower left and right panels, the principal connectivity gradient explaining most of the variance in the connectivity data was represented as separate layers. It usually follows the well-known axis of unimodal (e.g., visual, somatomotor networks; periphery; green-colored circles) to heteromodal (e.g., default mode network; core; red-colored circles) hierarchy of the brain.

relationship also led to changes in small-worldness, which is defined as the ratio between clustering coefficient (segregation) and characteristic path length (integration). In layman terms "small-worldness" can be regarded as the degree to which functionally clustered metropolitan cities (segregation) are connected with a small number of highways (integration). There is a debate regarding the relationship between smallworldness and post-stroke functional outcomes. Blaschke et al. ${ }^{47}$ demonstrated that acute stroke induces an abrupt increase in small-worldness across both humans and rodents, and the augmented small-worldness is significantly correlated with behavioral deficits. Van Meer et al. ${ }^{48}$ also indicated that smallworldness was increased when stroke was induced in mice. On the contrary, Siegel et al. ${ }^{36}$ reported that small-worldness decreased in patients with acute stroke. Since the results may differ depending on the lesion characteristics and timing of the evaluations ${ }_{1}^{36}$ further research is needed to reveal the effects of the complex interplay between segregation and integration on post-stroke changes in cognitive function.

\section{Hierarchy of network structures}

In addition to the concepts discussed above, taking into account the existence of a cortical hierarchy has proven impor- tant in understanding the effects of lesions on cognitive functions. It has been suggested that the composition of brain networks is not all at the same level, and some major axes form the brain's functional hierarchy (Figure 4). ${ }^{31}$

Cortical gradient analysis is a projection of the entire brain's connectivity to low-dimensional subspaces aimed at revealing the representative patterns of connectivity. ${ }^{49,50}$ In other words, the connectivity gradients reflect the underlying structure of functional similarity between cortical regions, and this approach has led to an understanding of the functional composition of the brain. ${ }^{31}$ The principal connectivity gradient, explaining most of the variance in connectivity data, usually follows the wellknown unimodal (e.g., visual, somatomotor networks which processes concrete information) to heteromodal (e.g., default mode network, which processing abstract information) hierarchy of the brain..$^{31,49}$ With these connectivity gradients as the reference axes, the macroscale change of brain network topologies, such as system segregation, can be explored. For example, one can analyze the distance between brain regions that were originally close enough in the gradient subspaces to be assigned the same canonical brain module in healthy controls. This measure is called the "dispersion" score, and the increase of dispersion after stroke suggests that the functional segregation of a 
network is presumably weakened due to pathological processes. ${ }^{51}$ Moreover, when the dispersion of frontoparietal, attention, and default mode networks were increased by aging, cognitive function also declined at the same time. ${ }^{50}$

Bayrak et al..$^{50}$ investigated the impact of ischemic stroke lesions on connectivity gradients. They reported that the degree of functional connectivity changes due to stroke lesions depends on the connectivity gradient, rather than the anatomical distance from the lesion. Functional alterations in closely related regions in the cortical gradient space were significantly associated with stroke severity and functional prognosis at discharge after stroke..$^{50}$

\section{Graphical representation: usefulness and limitations} To adequately evaluate aspects of these complex relationships, attempts are being made to not only represent them as a distinct parameter but also to visualize them as intuitive figures. These visualization methods enable us to identify the community structures of the brain and examine the spatial interactions of each brain region, such as core-periphery structures and hierarchical relationships (Figures 2 and 4). By calculating the strength of connectivity between each pair of nodes and representing it as a distance, it is possible to distinguish functionally independent major communities, which can be visualized through intuitive methods, such as spring-embedded figures (Figure 3). These methods can help understand the network structures, especially when evaluating longitudinal changes in one individual, ${ }^{41}$ or when comparing groups. Although these brain network visualization methods are also being used in individual patients, ${ }^{52}$ visualizing these spatial configurations carries the risk of misinterpretation and mathematical bias, due to the high-dimensional nature of neuroimaging data. Thus, graphical representations are still commonly used when evaluating differences between groups.

Recently, two approaches have been explored to overcome the limitations of the visualization process: (1) developing better dimensionality reduction methods to minimize bias and enhance interpretability, ${ }_{1}^{49}$ and (2) expanding the amount of data by increasing the acquisition time of rs-fMRI. ${ }^{35}$ First, there are many well-known linear dimensionality reduction methods, such as principal component analysis ${ }^{32,53}$ and independent component analysis. ${ }^{54}$ Furthermore, new nonlinear dimensionality reduction methods have recently been proposed to better reflect and represent nonlinear manifolds for data visualization, such as t-distributed stochastic neighbor embedding ${ }_{1}^{55}$ and uniform manifold approximation and projection. ${ }^{56}$ In addition, many different parcellations have been developed. ${ }^{57-60}$ Such dimensionality reduction methods should help to accu- rately visualize each individual patient's high-dimensional network structure with minimal bias. Moreover, they can help resolve multiple-comparison issues, improving the statistical power and sensitivity.

Second, the "precision functional mapping" approach utilizes a large amount of fMRI data from one individual, allowing us to obtain more robust features from individual's raw data and estimate more precise models of individualized functional brain networks. ${ }^{61,62}$ For example, a previous study acquired around 11 hours of rs-fMRI data per participant to examine precise functional brain architectures, and demonstrated that these functional brain connectomes are not only highly reliable within individual but also unique and distinct with each other. ${ }^{35}$ This approach has allowed the visualization of differences in the order and arrangement of each major node in individual patients. These visualization methods allow the identification of qualitative aspects of detailed network structures, which cannot be determined only by certain attributes, such as global efficiency, modularity, and system segregation. A recent study, using precision functional mapping, showed that the functional reorganization of the hand motor area consists of a specific remapping around the infarcted tissue after a large bilateral stroke. ${ }^{52}$ Despite these advantages, to ensure the reliability of the analysis, rs-fMRI images must be obtained for at least 30 minutes; thus, there are still limitations to the application of this method to patients with stroke in clinical practice, considering the cost and time involved. Once the individual functional mapping of each stroke patient becomes more reliable and reproducible, network analysis will be adopted in the clinical field as part of a precision medicine approach.

\section{Inference vs. observation: indirect and direct mapping}

Lesion-network mapping has recently attracted more and more attention. In most functional network studies, fMRIs were taken directly from patients and analyzed. ${ }^{32}$ For example, Siegel et al. ${ }^{32}$ obtained rs-fMRI data from more than 100 patients to confirm the correlation between each cognitive domain score and well-known RSNs. Although fMRI is a sophisticated anaIytic method, it is usually extremely difficult to obtain rs-fMRI data from patients, as fMRI requires limited movement for a long time in patients with stroke. Moreover, it is challenging to recruit a sufficient number of individuals for proper mathematical modeling.

To overcome these drawbacks, Fox et al. ${ }^{14}$ proposed a method called lesion-network mapping. This method uses a sufficient number of rs-fMRls from normal volunteers to create a 
normative functional connectivity map, and then overlaps the location of lesions in stroke patients with specific neurological symptoms to determine the network effects that are induced by each infarction. ${ }^{14}$ Several such studies applying this method have been recently published, ${ }^{63-65}$ along with reviews discussing the limitations of the process. . $^{13,66-68}$

The original purpose of the study by Darby et al.. ${ }^{63}$ Laganiere et al.. ${ }^{64}$ and Boes et al..$^{65}$ was to identify the neural substrates of peculiar neurological symptoms, which are difficult to identify by conventional lesion-symptom mapping methods, such as chorea and delusional misidentification. To this end, they focused on comparing patients with specific neurological symptoms and controls without those symptoms. The researchers also used the data of nearly 1,000 normal participants of the Brain Genomics Superstruct Project (GSP) to reconstruct normative connectivity maps. ${ }^{69}$ However, in the study by the Corbetta group, rs-fMRI images were taken directly in patients with stroke to create mathematical models predictive of cognitive test scores and verify their explanatory power. ${ }^{32,36}$

The Corbetta group ${ }^{13}$ showed that the lesion-network mapping was actually less predictive of cognitive prognosis than the direct functional imaging, structural connectivity imaging, and lesion location information itself. Thus, lesion-network mapping cannot replace the conventional methods based on data obtained directly from patients. ${ }^{13}$ They proved their argument using 7-T rs-fMRI data of 132 individuals from the $\mathrm{Hu}-$ man Connectome Project to reconstruct indirect lesion-network mapping, known as functional disconnectome, and comparing the prognostic power for PSCl using four methods: lesion information, structural disconnectome, direct functional disconnectome, and indirect functional disconnectome. In their results, the indirect lesion-network mapping showed the lowest predictive power for $\mathrm{PSCl}{ }^{13}$

In response, Cohen et al. ${ }^{66}$ conducted a study to evaluate the predictive power of the same four methods using different population-based and analytic approaches. In contrast to the study by Salvalaggio et al., ${ }^{13}$ the authors reconstructed the indirect functional disconnectome using 3-T MRI data from 1,000 individuals from the Human Connectome Project and focused on lesion location data. When the modeling was performed, applying the leave-one-out method and the "lesion mask" for each patient, the predictive power of the indirect functional disconnectome for behavioral outcomes showed an $R^{2}$ value similar to that of the lesion information-based approach.

The details of the technical points go beyond the scope of this review, but this debate demonstrates the recent developments and growing interest in analytic methods using rs-fMRI in the field of stroke. Upon further implementation, these meth- ods may become an important tool for easier screening, assessment, and identification of patients at high risk of PSCl, as well as facilitating research related to therapeutic intervention.

\section{Vulnerability}

So far, we have mainly considered the relationship between lesions and cognitive function. However, it is commonly observed that even similar-sized lesions occurring in similar locations may have different prognosis among patients. Therefore, further research is needed to elucidate the interactions between patients' underlying vulnerabilities and stroke lesions on network functions (Figure 5). Such vulnerabilities include the patient's age, sex, cognitive reserve, and comorbidities. Recent studies have worked on developing a new method that visualizes the underlying pathological processes, which are not observed with conventional images.

It is well known that amyloid pathology accelerates cognitive decline after stroke. Among patients with stroke, those with positive amyloid positron emission tomography (PET) results showed more distinct cognitive decline than those without them in a 3 -year follow-up study. ${ }^{70}$ This finding has been

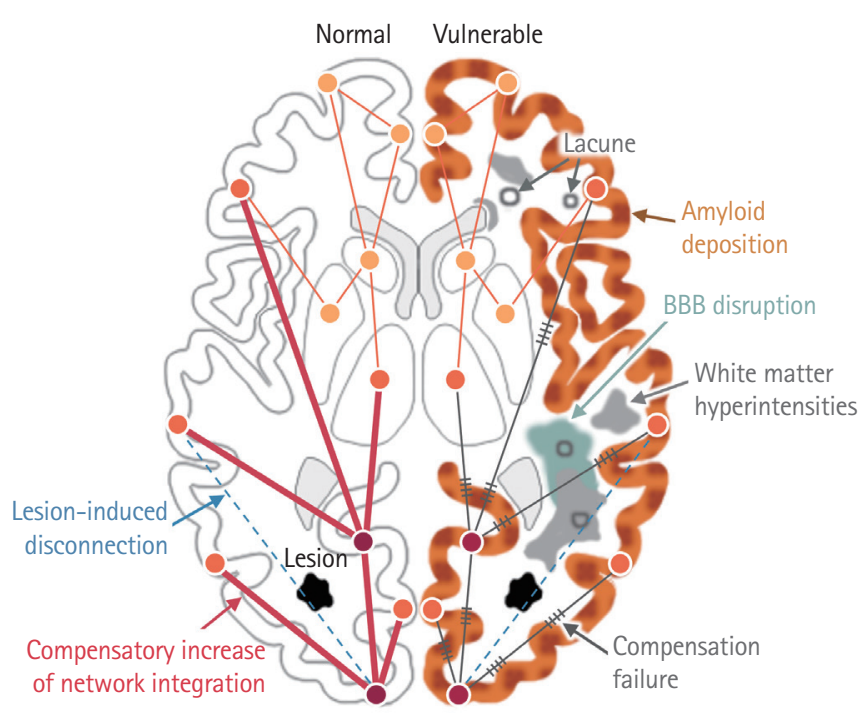

Figure 5. Effects of vulnerability factors on stroke-induced brain network changes. Prognosis after stroke can be affected by multiple risk factors, including amyloid decomposition, blood-brain barrier (BBB) disruption, and whiter matter hyperintensities. Left: Brain without vulnerability. Stroke lesion (black polygon) induces disconnection of brain regions (dashed skyblue line), followed by compensatory increase of brain network integration. Line thickness is proportional to connectivity strength. Circles indicate different brain regions. Right: Brain with vulnerability. Amyloid decomposition (brown speckles), white matter hyperintensities (gray polygon), BBB disruption (cyan polygon) disrupt compensatory brain network integration, indicated as gray line with hatch marks. Lacune are also marked as gray colored open circles. 
replicated through a longitudinal study in patients with subcortical vascular cognitive impairment, that had SVD without overt clinical stroke. ${ }^{11}$ However, some studies have reported that severe SVD is more important than amyloid pathology for long-term cognitive decline after stroke. ${ }^{72}$ Thus far, it seems clear that amyloid pathology has a detrimental effect on cognitive decline after stroke, although it is necessary to pay attention to the definition of the patient group, evaluation method, and follow-up period when interpreting these results. Furthermore, it has also been suggested that stroke itself alters amyloid PET findings, requiring caution in interpretation. In patients with recent infarction, the peri-infarct region had a relative increase in Pittsburgh compound B (PiB) retention compared with the contralateral mirror region, but no increase in global PiB retention. ${ }^{73}$ An animal study has shown a transitive increase in amyloid plaques around infarct areas, possibly reflecting the impairment of the amyloid clearance pathway around the infarcted tissue. ${ }^{74}$ Some researchers argue that such findings may be the result of free PiB leakage due to the BBB damage of the peri-infarct tissue, which requires careful interpretation. ${ }^{73}$ If the rapidly developing peripheral blood biomarkers for amyloid and tau are evaluated for this purpose, it will be possible to confirm the effect of these superimposed amyloid and tau pathology on cognitive impairment after stroke in a more multifaceted manner. ${ }^{75}$

BBB disruption has been studied in a variety of neurological disorders, including SVD, and several neurodegenerative diseases. However, BBB disruption cannot be easily evaluated using conventional imaging techniques. Thrippleton et al. ${ }^{76}$ visualized BBB leakage through dynamic contrast-enhanced MRI (DCE-MRI) in patients with lacunar infarction. Although the lesion identified by fluid-attenuated inversion recovery (FLAIR) was a small lacuna, DCE-MRI shows that ipsilesional hemispheres were accompanied by more extensive BBB changes. Further research is still needed to determine whether this change is a temporary phenomenon, how long it lasts, and whether it is related to functional prognosis; however, it has an important implication in terms of the underlying vulnerabilities of the patient's cognitive outcome, which would be difficult to identify based only on the lacunae number and location.

A recent study reported that a "free water" indicator of DTI showed a significant negative correlation with memory and frontal executive function even before the changes in fractional anisotropy (FA) and mean diffusivity (MD) appeared. It was suggested that the DTI free water may represent a subtle breakdown of the BBB..$^{77}$ DTI has the advantage of eliminating the need for a contrast medium, unlike DCE-MRI, and has been shown to reflect white matter pathology ahead of changes in
FA and white matter hyperintensities. ${ }^{78}$ DTI free water has also been reported to have increased in the substantia nigra of Parkinson's disease patients, as well as in the basal ganglia and thalamus of patients with other Parkinson syndromes, including multiple system atrophy and progressive supranuclear palsy. ${ }^{79}$ Because it can reflect various pathological mechanisms, further studies are needed to elucidate the exact relationship between DTI free water and BBB disruption. ${ }^{77}$

As mentioned earlier, it has been suggested that SVD is related to delayed cognitive decline after stroke. ${ }^{72}$ The severity of SVD is an important determinant for $\mathrm{PSCl}$, but the importance of the strategic location of SVD has also been suggested. ${ }^{19,80} \mathrm{~A}$ recently developed advanced imaging method, which can reflect the pathological changes of normal-appearing white matter (NAWM), is also expected to provide clarity regarding the vulnerability of patients after stroke. Cerebrovascular reactivity in the NAWM around leukoaraiosis was reduced, ${ }^{81}$ and BBB permeability was increased in the NAWM, prior to the development of white matter lesions. ${ }^{82}$ Microstructural alterations, reflected by high MD and low FA in DTI, were associated with poor processing speed and global cognition. ${ }^{83}$ Determining the extent of pathological processes in white matter regions prior to stroke is important for accurate prediction of cognitive prognosis and screening of patients for treatment. Another SVD marker is microinfarct, defined as a small lesion with less than $5 \mathrm{~mm}$ of T1 low and T2 high signal intensities in the cortical regions. ${ }^{84}$ van Veluw et al. ${ }^{84}$ verified in an autopsy study that these lesions, observed with 7-T MRI, were actually microinfarcts. These microinfarcts were more frequently found in patients with Alzheimer's disease than in healthy controls, and also showed a strong association with vascular dementia. ${ }^{85}$ Their presence was closely related to the deterioration of visuoconstructive and language functions, along with global cognition. It is noteworthy that these microinfarcts can also be observed with 3-T MRI. ${ }^{86}$ Microinfarcts were identified using $\mathrm{T} 2, \mathrm{~T} 1$, and FLAIR of 3-T MRI in 34 out of 231 stroke patients within 1 week (14.7\%) and significantly correlated with atrial fibrillation and confluent white matter hyperintensities. The authors suggested that microinfarcts may be related to microembolism and cerebral SVD. In terms of cognitive outcomes, they showed a significant correlation with visuospatial domain changes. In addition, perivascular spaces, cerebral microbleeds, and cerebral amyloid angiopathy are SVD markers that reflect the vulnerability of individual patients. ${ }^{87,88}$

\section{Challenges and future perspectives}

This is a field where significant progress has been made, but 
many outstanding challenges remain to be solved. In particular, the importance of cooperative research has recently emerged, as lesion-symptom mapping studies require a larger number of patients to provide wider lesion coverage. In addition, to investigate changes in the network structures while considering the diversity of stroke lesions and patient-specific characteristics, a large number of patients with well-described clinical features are needed. Multidisciplinary cooperation is also required as it impossible for clinicians to be familiar with all the sophisticated analysis methods that are being continuously developed.

Several representative international consortia have been recently formed to study the pathogenesis of PSCl. Among them, the Meta VCl Map obtained large amounts of lesion location information through 12 acute ischemic stroke cohorts, and also refined the lesion-symptom mapping analysis method. ${ }^{10}$ Stroke and Cognition Consortium (STROKOG) is another international consortium of longitudinal studies of PSCI that has presented important results from epidemiological and clinical studies of PSCl. ${ }^{89,90}$ The "Determinants of Incident Stroke Cognitive Outcomes and Vascular Effects on RecoverY (DISCOVERY)" is a consortium formed in North America, which plans to obtain multimodal data from over 8,000 patients, aiming to identify high-risk groups and the determinants of PSCl (ClinicalTrials. gov Identifier: NCT04916210).

fMRI and DTI are important research methods, but they have several drawbacks when applied to patients with stroke. These imaging techniques are burdensome for widespread implementation in patients with stroke, as they require a long scan time and are vulnerable to in-scanner movement. On the other hand, electroencephalography (EEG) is more affordable and easier to apply to patients; thus, it would be a good alternative for the study of network changes due to stroke, if reliable analysis techniques can be established for EEG.

The studies discussed in this review mainly focused on the pathophysiology of $\mathrm{PSCl}$, but their final aim was therapeutic intervention. In particular, research from a network perspective will provide important information on neuromodulation. Grefkes and Fink ${ }^{38}$ conducted fMRI imaging at various time points, i.e. as 1, 3, 7, and 90 days after stroke, and showed interesting results regarding the longitudinal changes in brain functional connectivity around the stroke lesion. In their study, activation of the contralateral homologous regions showed a suppressive effect in the early days after stroke onset, but a beneficial effect as time passed. This result has important implications for future neuromodulation targets and treatment windows. We do not yet know which stroke patients should be treated with neuromodulation and what is the optimal timing for the intervention. Further studies are required to provide critical information regarding this therapeutic intervention.

\section{Conclusions}

New imaging studies, as we have shown above, have increased knowledge on the patho-mechanisms of PSCl. Advances in classical lesion-symptom mapping techniques continue to reveal the localization of each cognitive domain in more sophisticated ways. In addition, novel methods have been implemented to comprehensively examine the disruption of functional and structural connections within the brain, caused by focal lesions, as well as the related changes in the brain system. Various graphical representations are useful to understand the detailed changes in network configuration induced by focal lesions. In addition, new information on the vulnerabilities that interfere with the recovery mechanism have been collected. International collaborations can deepen our understanding of the pathogenesis of PSCl and ultimately allow us to take a further step in the development of therapeutic interventions.

\section{Disclosure}

The authors have no financial conflicts of interest.

\section{Acknowledgments}

This research was supported by the National Research Foundation of Korea (NRF) grants funded by the Korea government, including 2017R1C1B5018132 (Ministry of Science and ICT; JaeSung Lim), IBS-R015-D1 (Institute for Basic Science; ChoongWan Woo), 2019R1C1C1004512 (National Research Foundation of Korea; Choong-Wan Woo), 2E30410-20-085 (KIST Institutional Program, Choong-Wan Woo), and 2018H1A2A1059844 (National Research Foundation of Korea; Jae-Joong Lee).

\section{References}

1. Broca P. Localisations des fonctions cérébrales. Siège de la faculté du langage articulé. Bull Soc Anthropol Paris 1863;4: 200-204.

2. Wernicke C. The symptom-complex of aphasia. In: Campbell TH. Disease of the Nervous System. London: Cassell, 1908;265 $-324$.

3. Ladino LD, Rizvi S, Téllez-Zenteno JF. The Montreal procedure: the legacy of the great Wilder Penfield. Epilepsy Behav 2018; 83:151-161.

4. Verdelho A, Wardlaw J, Pavlovic A, Pantoni L, Godefroy O, Du- 
ering $M$, et al. Cognitive impairment in patients with cerebrovascular disease: a white paper from the links between stroke ESO Dementia Committee. Eur Stroke J 2021;6:5-17.

5. Munsch F, Sagnier S, Asselineau J, Bigourdan A, Guttmann $C R$, Debruxelles $S$, et al. Stroke location is an independent predictor of cognitive outcome. Stroke 2016;47:66-73.

6. Duering $M$, Zieren $N$, Hervé $D$, Jouvent $E$, Reyes $S$, Peters $N$, et al. Strategic role of frontal white matter tracts in vascular cognitive impairment: a voxel-based lesion-symptom mapping study in CADASIL. Brain 2011;134(Pt 8):2366-2375.

7. Weaver NA, Zhao L, Biesbroek JM, Kuijf HJ, Aben HP, Bae HJ, et al. The Meta $\mathrm{VCl}$ Map consortium for meta-analyses on strategic lesion locations for vascular cognitive impairment using lesion-symptom mapping: design and multicenter pilot study. Alzheimers Dement (Amst) 2019;11:310-326.

8. Biesbroek JM, Lim JS, Weaver NA, Arikan G, Kang Y, Kim BJ, et al. Anatomy of phonemic and semantic fluency: a lesion and disconnectome study in 1231 stroke patients. Cortex 2021; 143:148-163.

9. Biesbroek JM, van Zandvoort MJ, Kuijf HJ, Weaver NA, Kappelle $\sqcup$, Vos PC, et al. The anatomy of visuospatial construction revealed by lesion-symptom mapping. Neuropsychologia 2014;62:68-76.

10. Weaver NA, Kuijf HJ, Aben HP, Abrigo J, Bae HJ, Barbay M, et al. Strategic infarct locations for post-stroke cognitive impairment: a pooled analysis of individual patient data from 12 acute ischaemic stroke cohorts. Lancet Neurol 2021;20:448459.

11. Hillis $A E$, Beh $Y Y$, Sebastian R, Breining B, Tippett DC, Wright $A$, et al. Predicting recovery in acute poststroke aphasia. Ann Neurol 2018;83:612-622.

12. Weaver NA, Kancheva AK, Lim JS, Biesbroek JM, Wajer IMH, Kang $Y$, et al. Post-stroke cognitive impairment on the MiniMental State Examination primarily relates to left middle cerebral artery infarcts. Int J Stroke 2021 Jan 20 [Epub]. https:// doi.org/10.1177/1747493020984552.

13. Salvalaggio A, De Filippo De Grazia M, Zorzi M, Thiebaut de Schotten M, Corbetta M. Post-stroke deficit prediction from lesion and indirect structural and functional disconnection. Brain 2020;143:2173-2188.

14. Fox MD. Mapping symptoms to brain networks with the human connectome. N Engl J Med 2018;379:2237-2245.

15. Carrera $\mathrm{E}_{1}$ Tononi G. Diaschisis: past, present, future. Brain 2014;137(Pt 9):2408-2422.

16. Duering $M$, Righart $R$, Csanadi $E_{1}$ Jouvent $E_{1}$ Hervé $D_{1}$ Chabriat $H_{\text {, et }}$ al. Incident subcortical infarcts induce focal thinning in connected cortical regions. Neurology 2012;79:2025-2028.

17. Alstott J, Breakspear M, Hagmann P, Cammoun L, Sporns 0.
Modeling the impact of lesions in the human brain. PLoS Comput Biol 2009;5:e1000408.

18. van den Heuvel MP, Sporns 0. Rich-club organization of the human connectome. J Neurosci 2011;31:15775-15786.

19. Lim JS, Kim N, Jang MU, Han MK, Kim S, Baek MJ, et al. Cortical hubs and subcortical cholinergic pathways as neural substrates of poststroke dementia. Stroke 2014;45:1069-1076.

20. Griffis JC, Metcalf NV, Corbetta M, Shulman GL. Structural disconnections explain brain network dysfunction after stroke. Cell Rep 2019;28:2527.e9-2540.e9.

21. Shen J, Tozer DJ, Markus HS, Tay J. Network efficiency mediates the relationship between vascular burden and cognitive impairment: a diffusion tensor imaging study in UK Biobank. Stroke 2020;51:1682-1689.

22. Thiebaut de Schotten M, Foulon C, Nachev P. Brain disconnections link structural connectivity with function and behaviour. Nat Commun 2020;11:5094.

23. Greicius MD, Krasnow B, Reiss AL, Menon V. Functional connectivity in the resting brain: a network analysis of the default mode hypothesis. Proc Natl Acad Sci U S A 2003;100: 253-258.

24. Bressler SL, Menon V. Large-scale brain networks in cognition: emerging methods and principles. Trends Cogn Sci 2010; 14:277-290.

25. Greicius M. Resting-state functional connectivity in neuropsychiatric disorders. Curr Opin Neurol 2008;21:424-430.

26. Lee JJ, Kim HJ, Čeko M, Park BY, Lee SA, Park H, et al. A neuroimaging biomarker for sustained experimental and clinical pain. Nat Med 2021;27:174-182.

27. Sporns 0. Contributions and challenges for network models in cognitive neuroscience. Nat Neurosci 2014;17:652-660.

28. Farahani FV, Karwowski W, Lighthall NR. Application of graph theory for identifying connectivity patterns in human brain networks: a systematic review. Front Neurosci 2019;13:585.

29. Matthews PM, Hampshire A. Clinical concepts emerging from fMRI functional connectomics. Neuron 2016;91:511-528.

30. Dacosta-Aguayo R, Graña, M, Iturria-Medina Y, Fernández-

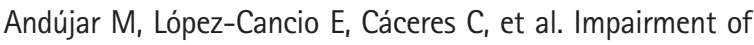
functional integration of the default mode network correlates with cognitive outcome at three months after stroke. Hum Brain Mapp 2015;36:577-590.

31. Mesulam MM. From sensation to cognition. Brain 1998;121 (Pt 6):1013-1052.

32. Siegel JS, Ramsey LE, Snyder AZ, Metcalf NV, Chacko RV, Weinberger $K_{1}$ et al. Disruptions of network connectivity predict impairment in multiple behavioral domains after stroke. Proc Natl Acad Sci U S A 2016;113:E4367-E4376.

33. Rubinov M, Sporns 0. Complex network measures of brain 
connectivity: uses and interpretations. Neuroimage 2010;52: 1059-1069.

34. Bullmore E, Sporns 0. Complex brain networks: graph theoretical analysis of structural and functional systems. Nat Rev Neurosci 2009;10:186-198.

35. Gordon EM, Laumann TO, Gilmore AW, Newbold DJ, Greene DJ, Berg JJ, et al. Precision functional mapping of individual human brains. Neuron 2017;95:791.e7-807.e7.

36. Siegel JS, Seitzman BA, Ramsey LE, Ortega M, Gordon EM, Dosenbach NUF, et al. Re-emergence of modular brain networks in stroke recovery. Cortex 2018;101:44-59.

37. Wig GS. Segregated systems of human brain networks. Trends Cogn Sci 2017;21:981-996.

38. Grefkes C, Fink GR. Connectivity-based approaches in stroke and recovery of function. Lancet Neurol 2014;13:206-216.

39. Gratton C, Nomura EM, Pérez F, D'Esposito M. Focal brain lesions to critical locations cause widespread disruption of the modular organization of the brain. J Cogn Neurosci 2012;24: 1275-1285.

40. Tao Y, Rapp B. The effects of lesion and treatment-related recovery on functional network modularity in post-stroke dysgraphia. Neuroimage Clin 2019;23:101865.

41. Chan MY, Park DC, Savalia NK, Petersen SE, Wig GS. Decreased segregation of brain systems across the healthy adult lifespan. Proc Natl Acad Sci U S A 2014;111:E4997-E5006.

42. Nomura EM, Gratton C, Visser RM, Kayser A, Perez F, D'Esposito M. Double dissociation of two cognitive control networks in patients with focal brain lesions. Proc Natl Acad SciUSA 2010;107:12017-12022.

43. Aben HP, Biessels GJ, Weaver NA, Spikman JM, Visser-Meily $J M A$, de Kort PLM, et al. Extent to which network hubs are affected by ischemic stroke predicts cognitive recovery. Stroke 2019;50:2768-2774.

44. Guo J, Biswal BB, Han S, Li J, Yang S, Yang M, et al. Altered dynamics of brain segregation and integration in poststroke aphasia. Hum Brain Mapp 2019;40:3398-3409.

45. Wang Y, Wang C, Miao P, Liu J, Wei Y, Wu L, et al. An imbalance between functional segregation and integration in patients with pontine stroke: a dynamic functional network connectivity study. Neuroimage Clin 2020;28:102507.

46. Xu X, Tang R, Zhang L, Cao Z. Altered topology of the structural brain network in patients with post-stroke depression. Front Neurosci 2019;13:776.

47. Blaschke SJ, Hensel L, Minassian A, Vlachakis S, Tscherpel C, Vay SU, et al. Translating functional connectivity after stroke: functional magnetic resonance imaging detects comparable network changes in mice and humans. Stroke 2021;52:29482960.
48. van Meer MP, Otte WM, van der Marel K, Nijboer CH, Kavelaars A, van der Sprenkel JW, et al. Extent of bilateral neuronal network reorganization and functional recovery in relation to stroke severity. J Neurosci 2012;32:4495-4507.

49. Margulies DS, Ghosh SS, Goulas A, Falkiewicz M, Huntenburg $J M$, Langs $G$, et al. Situating the default-mode network along a principal gradient of macroscale cortical organization. Proc Natl Acad Sci U S A 2016;113:12574-12579.

50. Bayrak Ş, Khalil AA, Villringer K, Fiebach JB, Villringer A, Margulies DS, et al. The impact of ischemic stroke on connectivity gradients. Neuroimage Clin 2019;24:101947.

51. Bethlehem RAI, Paquola C, Seidlitz J, Ronan L, Bernhardt B, Consortium CC, et al. Dispersion of functional gradients across the adult lifespan. Neuroimage 2020;222:117299.

52. Laumann TO, Ortega M, Hoyt CR, Seider NA, Snyder AZ, Dosenbach NU, et al. Brain network reorganisation in an adolescent after bilateral perinatal strokes. Lancet Neurol 2021; 20:255-256.

53. Ramsey LE, Siegel JS, Lang CE, Strube M, Shulman GL, Corbetta $\mathrm{M}$. Behavioural clusters and predictors of performance during recovery from stroke. Nat Hum Behav 2017;1:0038.

54. Specht K, Zahn R, Willmes K, Weis S, Holtel C, Krause BJ, et al. Joint independent component analysis of structural and functional images reveals complex patterns of functional reorganisation in stroke aphasia. Neuroimage 2009;47:20572063.

55. Hu Y, Li X, Wang L, Han B, Nie S. T-distribution stochastic neighbor embedding for fine brain functional parcellation on rs-fMRI. Brain Res Bull 2020;162:199-207.

56. Bahrami M, Lyday RG, Casanova R, Burdette JH, Simpson SL, Laurienti PJ. Using low-dimensional manifolds to map relationships between dynamic brain networks. Front Hum Neurosci 2019;13:430.

57. Yourganov G, Fridriksson J, Rorden C, Gleichgerrcht E, Bonilha L. Multivariate connectome-based symptom mapping in poststroke patients: networks supporting language and speech. $J$ Neurosci 2016;36:6668-6679.

58. De Baene W, Rijnen SJM, Gehring K, Meskal I, Rutten GM, Sitskoorn MM. Lesion symptom mapping at the regional level in patients with a meningioma. Neuropsychology 2019;33: 103-110.

59. Thothathiri M, Kimberg DY, Schwartz MF. The neural basis of reversible sentence comprehension: evidence from voxelbased lesion symptom mapping in aphasia. J Cogn Neurosci 2012;24:212-222.

60. Rondina JM, Filippone M, Girolami M, Ward NS. Decoding post-stroke motor function from structural brain imaging. Neuroimage Clin 2016;12:372-380. 
61. Newbold DJ, Laumann TO, Hoyt CR, Hampton JM, Montez DF, Raut RV, et al. Plasticity and spontaneous activity pulses in disused human brain circuits. Neuron 2020;107:580.e6-589. e6.

62. Greene DJ, Marek S, Gordon EM, Siegel JS, Gratton C, Laumann TO, et al. Integrative and network-specific connectivity of the basal ganglia and thalamus defined in individuals. Neuron 2020;105:742.e6-758.e6.

63. Darby RR, Laganiere S, Pascual-Leone A, Prasad S, Fox MD. Finding the imposter: brain connectivity of lesions causing delusional misidentifications. Brain 2017;140:497-507.

64. Laganiere $S$, Boes $A D$, Fox MD. Network localization of hemichorea-hemiballismus. Neurology 2016;86:2187-2195.

65. Boes AD, Prasad S, Liu H, Liu O, Pascual-Leone A, Caviness VS $J r$, et al. Network localization of neurological symptoms from focal brain lesions. Brain 2015;138(Pt 10):3061-3075.

66. Cohen AL, Ferguson MA, Fox MD. Lesion network mapping predicts post-stroke behavioural deficits and improves localization. Brain 2021;144:e35.

67. Cohen AL, Fox MD. Reply: the influence of sample size and arbitrary statistical thresholds in lesion-network mapping. Brain 2020;143:e41.

68. Salvalaggio A, Pini L, De Filippo De Grazia M, Thiebaut De Schotten M, Zorzi M, Corbetta M. Reply: lesion network mapping: where do we go from here? Brain 2021;144:e6.

69. Holmes AJ, Hollinshead MO, O'Keefe TM, Petrov VI, Fariello GR, Wald LL, et al. Brain Genomics Superstruct Project initial data release with structural, functional, and behavioral measures. Sci Data 2015:2:150031.

70. Liu W, Wong A, Au L, Yang J, Wang Z, Leung EY, et al. Influence of amyloid-beta on cognitive decline after stroke/transient ischemic attack: three-year longitudinal study. Stroke 2015;46:3074-3080.

71. Ye BS, Seo SW, Kim JH, Kim GH, Cho H, Noh Y, et al. Effects of amyloid and vascular markers on cognitive decline in subcortical vascular dementia. Neurology 2015;85:1687-1693.

72. Mok VCT, Lam BYK, Wang Z, Liu W, Au L, Leung EYL, et al. Delayed-onset dementia after stroke or transient ischemic attack. Alzheimers Dement 2016;12:1167-1176.

73. Ly JV, Rowe CC, Villemagne VL, Zavala JA, Ma H, Sahathevan $R$, et al. Subacute ischemic stroke is associated with focal $11 \mathrm{C}$ $\mathrm{PiB}$ positron emission tomography retention but not with global neocortical Abeta deposition. Stroke 2012;43:13411346.

74. Garcia-Alloza M, Gregory J, Kuchibhotla KV, Fine S, Wei Y, Ayata $C_{1}$ et al. Cerebrovascular lesions induce transient betaamyloid deposition. Brain 2011;134(Pt 12):3697-3707.

75. Toombs J, Zetterberg $\mathrm{H}$. In the blood: biomarkers for amyloid pathology and neurodegeneration in Alzheimer's disease. Brain Commun 2020;2:fcaa054.

76. Thrippleton MJ, Backes WH, Sourbron $S_{1}$ Ingrisch $M$, van Osch MJP, Dichgans $M$, et al. Quantifying blood-brain barrier leakage in small vessel disease: review and consensus recommendations. Alzheimers Dement 2019;15:840-858.

77. Maillard , $_{\text {, Fletcher }}$, Singh B, Martinez O, Johnson DK, Olichney JM, et al. Cerebral white matter free water: a sensitive biomarker of cognition and function. Neurology 2019;92: e2221-e2231.

78. Maillard P, Mitchell GF, Himali JJ, Beiser A, Fletcher E, Tsao $C W$, et al. Aortic stiffness, increased white matter free water, and altered microstructural integrity: a continuum of injury. Stroke 2017;48:1567-1573.

79. Planetta PJ, Ofori E, Pasternak O, Burciu RG, Shukla P, DeSimone $\mathrm{JC}$, et al. Free-water imaging in Parkinson's disease and atypical parkinsonism. Brain 2016;139(Pt 2):495-508.

80. Bocti C, Swartz RH, Gao FQ, Sahlas DJ, Behl P, Black SE. A new visual rating scale to assess strategic white matter hyperintensities within cholinergic pathways in dementia. Stroke 2005;36:2126-2131.

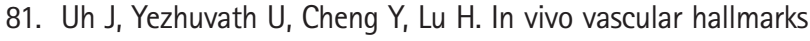
of diffuse leukoaraiosis. J Magn Reson Imaging 2010;32:184190.

82. Topakian R, Barrick TR, Howe FA, Markus HS. Blood-brain barrier permeability is increased in normal-appearing white matter in patients with lacunar stroke and leucoaraiosis. $J$ Neurol Neurosurg Psychiatry 2010;81:192-197.

83. Vernooij MW, Ikram MA, Vrooman HA, Wielopolski PA, Krestin GP, Hofman $A$, et al. White matter microstructural integrity and cognitive function in a general elderly population. Arch Gen Psychiatry 2009;66:545-553.

84. van Veluw SJ, Zwanenburg JJ, Engelen-Lee J, Spliet WG, Hendrikse J, Luijten PR, et al. In vivo detection of cerebral cortical microinfarcts with high-resolution 7T MRI. J Cereb Blood Flow Metab 2013;33:322-329.

85. van Veluw SJ, Shih AY, Smith EE, Chen C, Schneider JA, Wardlaw JM, et al. Detection, risk factors, and functional consequences of cerebral microinfarcts. Lancet Neurol 2017;16: 730-740.

86. Wang Z, van Veluw SJ, Wong A, Liu W, Shi L, Yang J, et al. Risk factors and cognitive relevance of cortical cerebral microinfarcts in patients with ischemic stroke or transient ischemic attack. Stroke 2016;47:2450-2455.

87. Wardlaw JM, Smith EE, Biessels GJ, Cordonnier C, Fazekas F, Frayne $R$, et al. Neuroimaging standards for research into small vessel disease and its contribution to ageing and neurodegeneration. Lancet Neurol 2013;12:822-838. 
88. Smith $E E$, Beaudin AE. New insights into cerebral small vessel disease and vascular cognitive impairment from MRI. Curr Opin Neurol 2018;31:36-43.

89. Lo JW, Crawford JD, Desmond DW, Godefroy O, Jokinen $H_{1}$ Mahinrad $S$, et al. Profile of and risk factors for poststroke cognitive impairment in diverse ethnoregional groups. Neu- rology 2019;93:e2257-e2271.

90. Lo JW, Crawford JD, Samaras K, Desmond DW, Köhler S, Staals $J$, et al. Association of prediabetes and type 2 diabetes with cognitive function after stroke: a STROKOG Collaboration Study. Stroke 2020;51:1640-1646. 